\title{
Exercise: the brittle cornerstone of type 2 diabetes treatment
}

\author{
S. F. E. Praet • L. J. C. van Loon
}

Received: 27 July 2007 / Accepted: 7 December 2007 / Published online: 9 January 2008

(C) The Author(s) 2007

\begin{abstract}
Regular exercise has been recommended for diabetes patients for many years; however, it is not widely used clinically. This may be because of high costs, lack of reimbursement, low compliance and/or absence of proper infrastructure. Alternatively, structured exercise therapy may be underutilised because current guidelines do not include detailed information on the preferred type and intensity of exercise that should be applied to maximise the benefits of exercise for different subgroups of patients with type 2 diabetes. Based on available evidence and our own clinical research experience this article proposes that exercise therapy in type 2 diabetes might be more effective if (1) cardiac rehabilitation programmes served as a model for 'pre-cardiac diabetes rehabilitation'; (2) resistance exercise were prescribed for sarcopenic or severely deconditioned type 2 diabetes patients; and (3) a multidisciplinary approach and continued exercise training under personal supervision became standard therapy. Nevertheless, more clinical research is warranted to establish the efficacy of an approach that takes into account type 2 diabetes subpopulations at different stages of the disease and with different levels of comorbidity.
\end{abstract}

Keywords Anxiety - Depression · Exercise - Injury risk · Osteoarthritis · Prescription · Rehabilitation $\cdot$ Review . Sarcopenia $\cdot$ Type 2 diabetes mellitus

S. F. E. Praet $(\bowtie) \cdot$ L. J. C. van Loon

Nutrition and Toxicology Research Institute Maastricht (NUTRIM), Department of Human Movement Sciences, Maastricht University,

Universiteitssingel 50,

6229 ER Maastricht, the Netherlands

e-mail: Stephan.Praet@BW.unimaas.nl

\begin{abstract}
Abbreviations
DARE Diabetes Aerobic and Resistance Exercise
\end{abstract}

\section{Introduction}

Together with medication and diet, exercise is considered one of the three cornerstones of diabetes care programmes. Despite a substantial body of evidence showing the benefits of exercise, its clinical application is still underutilised. Although medical doctors generally consider it the patient's personal responsibility to increase physical activity levels, physicians and healthcare workers tend to underestimate their potential role in changing the diabetes patient's lifestyle. Moreover, from both a sports physician's and exercise scientist's perspective, current exercise programmes and clinical guidelines are too general to allow an exercise intervention programme to be tailor made for the individual diabetes patient. The goal of this article is to revive the discussion on the clinical application of exercise in the treatment of type 2 diabetes and to provide general practitioners and endocrinologists with a more specific framework to optimise exercise therapy.

\section{Lessons learned from cardiac rehabilitation}

Van Noorden and Isaak identified regular exercise, along with diet and insulin, as one of the three components of good therapy as early as 1927 (for references, see [1]). From a physiological perspective, structured exercise interventions have been shown to be at least as efficacious as the pharmaceutical agents currently available for improving glycaemic control $[2,3]$ and cardiovascular risk 
profile [4]. Despite the growing body of evidence showing the health benefits of exercise in type 2 diabetes, a recently published large-scale US survey shows that the majority of patients with diabetes do not engage in regular physical activity [5], and there is currently no reason to believe that this is any different across the rest of the world. Although physician advice has been shown to be a strong predictor of attempts to change lifestyle habits, health professionals may not take the time or provide enough specific information to help patients successfully change their physical activity behaviour [6]. Apparently, most physicians and diabetes nurses around the world find it difficult to prescribe structured exercise routines for individual patients, and the high costs, lack of reimbursement, low compliance and/or absence of proper infrastructure may be responsible for this [7]. Not so long ago, cardiac patients and their doctors experienced similar problems; however, exercise-based cardiac rehabilitation programmes have been shown to be effective and feasible if available evidence-based guidelines are supported by a motivated and knowledgeable staff and applied in a patient-tailored way [8]. Although the longterm outcome of such an approach is currently being investigated in a large-scale prospective study on intensive lifestyle interventions, the preliminary results look promising [9]. Thus, cardiac rehabilitation programmes could serve as a model for future 'pre-cardiac diabetes rehabilitation' programmes.

\section{Do the current diabetes treatment guidelines need to be extended?}

Current guidelines from the American Diabetes Association recognise the therapeutic strength of exercise intervention [10] and prescribe an exercise frequency of at least three times per week with no more than two consecutive days without physical activity. However, these clinical guidelines need to be considered as a minimum prescription and, therefore, as a less than optimum situation. Recent guidelines on physical activity and public health in older adults from the American College of Sports Medicine and American Heart Association also recommend musclestrengthening and balance exercise as an integrated part of physical activity intervention in older adults [11]. Older adults with chronic conditions, including coronary artery disease, osteoarthritis and type 2 diabetes, should develop an activity plan in consultation with a healthcare provider, taking into account therapeutic and risk management issues [11]. Long-standing insulin-treated type 2 diabetic patients form an expanding subpopulation and require a more individualised approach. These type 2 diabetic patients generally suffer from severe exercise intolerance as a result of low oxidative capacity, neuropathy-related muscle weakness, sarcopenia and/or micro- and macrovascular disease [12]. As generic exercise intervention programmes are too demanding for most of these patients, intermediate programmes are needed to bring the patient to a level at which they are able to participate in such programmes. The intermediate programmes should involve bouts of relatively high-intensity exercise, applied in an intermittent fashion, to augment muscle strength and functional performance. The greater amount of work performed and lower cardiorespiratory load achieved with intermittent exercise $[13,14]$ not only allows for a greater metabolic adaptative response in healthy individuals [15], but has also proved to be more effective than endurance exercise training in patients experiencing pulmonary limitations [14]. Although these so-called 'in-and-out' exercises have been proved safe in both cardiac [13] and high-risk insulin-treated type 2 diabetic patients [16], the long-term efficacy of such intermediate programmes in type 2 diabetes patients remains to be established.

\section{Current lifestyle interventions place an emphasis on obesity}

Most lifestyle intervention programmes generally focus on the implementation of endurance exercise. Historically, this type of exercise has been associated with efficacy in reducing body fat mass and improving aerobic fitness and whole-body insulin sensitivity. An endurance exercise programme should involve energy expenditure equivalent to $\sim 1.7-2.1 \mathrm{MJ}(\approx 400-500 \mathrm{kcal})$ per exercise bout on 3 , but preferably 5 days per week $[17,18]$. More vigorous exercise in uncomplicated insulin-resistant states will further improve glycaemic control and enhance cardiorespiratory fitness and microvascular function. Recently diagnosed obese type 2 diabetic patients would benefit most from a programme mainly consisting of moderateintensity endurance exercise, aiming for a minimum energy expenditure of at least 5 MJ $(\approx 1,200 \mathrm{kcal})$ [18], but preferably more than $8.5 \mathrm{MJ}(\approx 2,000 \mathrm{kcal})$, per week [19]. In the obese type 2 diabetic patient such an endurance intervention programme should be combined with dietary restriction to maximise fat mass loss. Given the concomitant risk of losing muscle mass, at least one resistance exercise session should be included per week [20]. However, it remains difficult to translate laboratory results to community settings [21], and long-term outcomes may be disappointing. Therefore, adequate intensity, progression and adherence are required, or benefits will not accrue or persist. 


\section{Greater focus on resistance exercise}

Both endurance and resistance exercise have been shown to improve whole-body insulin sensitivity and/or glucose tolerance $[3,22]$. It was recently shown that both endurance and resistance exercise have equal therapeutic strength in insulin-resistant states $[3,22]$. The combined application of endurance and resistance exercise training might be of even greater clinical benefit in patients with uncomplicated type 2 diabetes [3]. However, the combined exercise benefits seen in the Diabetes Aerobic and Resistance Exercise (DARE) trial may, at least in part, be explained by a higher weekly energy expenditure. Nevertheless, for several other reasons, resistance exercise represents an attractive exercise mode to prescribe and implement in therapeutic intervention programmes. Unlike the DARE study population with uncomplicated type 2 diabetes, the majority of type 2 diabetic patients suffer from some form of muscle weakness, osteoarthritis, mobility impairments, peripheral and/or cardiovascular disease, not only resulting in reduced exercise tolerance [23], but also in a potentially high risk of overload injuries [3, Praet et al., unpublished results]. In line with this, it has proved difficult to get type 2 diabetic patients to adhere to strict endurance exercise intervention routines [7]. Alternatively, moderate intensity endurance exercise combined with resistance (i.e. intermittent intensity) exercise represents a lower cardiovascular and cardiorespiratory challenge and improves functional performance to a similar extent [13, 14]. Although we have recently shown that long-term adherence in such a primary healthcarebased exercise intervention is $\sim 40 \%$ (Praet et al., unpublished results), the combination of endurance and resistance type exercise is preferred as it makes the programme more varied and, as such, increases the adherence and compliance rates.

\section{Aging, sarcopenia and type 2 diabetes}

A similar approach with even greater emphasis on resistance exercise should be particularly useful in the vastly expanding subgroup of elderly ( $>70$ years) type 2 diabetic patients [24]. Loss of skeletal muscle mass represents one of the main factors responsible for the increase in the incidence of type 2 diabetes with age [25]. The loss of muscle mass is proportionally related to the reduction in blood glucose disposal capacity and the decline in muscle strength [24, 26]. The latter prevents many elderly diabetic patients from participating in lifestyle intervention programmes [24]. Therefore, effective exercise intervention programmes targeting insulin resistance in elderly type 2 diabetes patients should focus on increasing skeletal muscle mass and strength - therapeutic advice that has been well summarised by the slogan, 'Bring on the heavy weights' [24]. They should consist mainly of resistance exercise, with exercise volume and intensity being progressively increased towards three sets of between eight and ten repetitions, with intensities ranging from 50 $80 \%$ of one repetition maximum (1-RM) for between seven and ten exercises and/or muscle groups [22]. Adequate nutritional support might further improve the benefits of resistance type exercise training in elderly patients [27].

\section{Complex diabetes patients require more dedicated diabetes care teams}

When prescribing exercise intervention for type 2 diabetes patients it should be noted that long-term adherence to an exercise intervention programme may vary with the presence of comorbidities, deconditioning and/or orthopaedic limitations [26]. Before exposing type 2 diabetes patients to more vigorous exercise programmes, silent myocardial ischaemia should be excluded if their cardiac risk over 10 years exceeds $10 \%$ [10]. However, patients with higher anxiety and/or depression scores also perceive more barriers to exercise [28]. Therefore, a multidisciplinary team should not only consider the level of impairment to exercise tolerance, but also appraise psychosocial stress factors. Such an individually tailored exercise intervention programme may reduce the risk of dropout and empower the patient to adhere to such lifestyle changes [29].

\section{Conclusions and future research}

Clinical implementation of exercise in the treatment of type 2 diabetes is important for the primary and secondary prevention of type 2 diabetes, but is still far from being standard practice. To enhance its clinical application, lessons may be learned from the experience with exercisebased cardiac rehabilitation programmes. Moreover, current guidelines should include more detailed information on the preferred type and intensity of exercise for specific patient subgroups. Before choosing the most appropriate exercise modalities, the patient's disease stage should be well characterised and exercise stress testing should be considered. Based on baseline aerobic fitness, level of comorbidity and appendicular skeletal muscle mass and strength, patients should be provided with a fitting exercise intervention programme to optimise its therapeutic value. Most type 2 diabetes patients find it difficult to adhere to a structured exercise intervention regimen, and compliance 
can be enhanced by proper multidisciplinary care and continued exercise training under strict personal supervision. More clinical research is warranted to establish the efficacy of a more differentiated approach for type 2 diabetes subpopulations at different stages of the disease and with different levels of comorbidity.

Acknowledgements The authors' salaries are funded by research grants from the Dutch Ministry of Health, Welfare and Sport (S. F. E. Praet) and Maastricht University (L. J. C. van Loon). The funding agencies have played no role in the submission and/or preparation of this manuscript.

Duality of interest The authors declare that there is no duality of interest associated with this manuscript.

Open Access This article is distributed under the terms of the Creative Commons Attribution Noncommercial License which permits any noncommercial use, distribution, and reproduction in any medium, provided the original author(s) and source are credited.

\section{References}

1. Katsch D (1939) Work therapy for diabetes patients. [Die Arbeitstherapie der Zuckerkranken.]. Erg Physikal Diat Ther 1:1-36

2. Conn VS, Hafdahl AR, Mehr DR, Lemaster JW, Brown SA, Nielsen PJ (2007) Metabolic effects of interventions to increase exercise in adults with type 2 diabetes. Diabetologia 50:913-921

3. Sigal RJ, Kenny GP, Boule NG et al (2007) Effects of aerobic training, resistance training, or both on glycemic control in type 2 diabetes: a randomized trial. Ann Intern Med 147:357-369

4. Di Loreto C, Fanelli C, Lucidi P et al (2005) Make your diabetic patients walk: long-term impact of different amounts of physical activity on type 2 diabetes. Diabetes Care 28:1295-1302

5. Morrato EH, Hill JO, Wyatt HR, Ghushchyan V, Sullivan PW (2007) Physical activity in U.S. adults with diabetes and at risk for developing diabetes, 2003. Diabetes Care 30:203-209

6. Morrato EH, Hill JO, Wyatt HR, Ghushchyan V, Sullivan PW (2006) Are health care professionals advising patients with diabetes or at risk for developing diabetes to exercise more? Diabetes Care 29:543-548

7. Thomas N, Alder E, Leese GP (2004) Barriers to physical activity in patients with diabetes. Postgrad Med J 80:287-291

8. Taylor RS, Brown A, Ebrahim S et al (2004) Exercise-based rehabilitation for patients with coronary heart disease: systematic review and meta-analysis of randomized controlled trials. Am J Med 116:682-692

9. Pi-Sunyer X, Blackburn G, Brancati FL et al (2007) Reduction in weight and cardiovascular disease risk factors in individuals with type 2 diabetes: one-year results of the look AHEAD trial. Diabetes Care 30:1374-1383

10. American Diabetes Association (2007) Standards of medical care in diabetes-2007. Diabetes Care 30(1):S4-S41

11. Nelson ME, Rejeski WJ, Blair SN et al (2007) Physical activity and public health in older adults: recommendation from the American College of Sports Medicine and the American Heart Association. Circulation 116:1094-1105
12. Fang ZY, Sharman J, Prins JB, Marwick TH (2005) Determinants of exercise capacity in patients with type 2 diabetes. Diabetes Care 28:1643-1648

13. Meyer K, Lehmann M, Sunder G, Keul J, Weidemann H (1990) Interval versus continuous exercise training after coronary bypass surgery: a comparison of training-induced acute reactions with respect to the effectiveness of the exercise methods. Clin Cardiol 13:851-861

14. Sabapathy S, Kingsley RA, Schneider DA, Adams L, Morris NR (2004) Continuous and intermittent exercise responses in individuals with chronic obstructive pulmonary disease. Thorax 59:1026-1031

15. Burgomaster KA, Howarth KR, Phillips SM et al (2008) Similar metabolic adaptations during exercise after low volume sprint interval and traditional endurance training in humans. J Physiol 586: $151-160$

16. De Feyter HM, Praet SF, van den Broek NM et al (2007) Exercise training improves glycemic control in long-standing, insulintreated type 2 diabetes patients. Diabetes Care 30:2511-2513

17. O'Donovan G, Kearney EM, Nevill AM, Woolf-May K, Bird SR (2005) The effects of 24 weeks of moderate- or high-intensity exercise on insulin resistance. Eur J Appl Physiol 95:522-528

18. Houmard JA, Tanner CJ, Slentz CA, Duscha BD, McCartney JS, Kraus WE (2004) Effect of the volume and intensity of exercise training on insulin sensitivity. J Appl Physiol 96:101-106

19. Helmrich SP, Ragland DR, Leung RW, Paffenbarger RS Jr. (1991) Physical activity and reduced occurrence of non-insulin-dependent diabetes mellitus. N Engl J Med 325:147-152

20. Daly RM, Dunstan DW, Owen N, Jolley D, Shaw JE, Zimmet PZ (2005) Does high-intensity resistance training maintain bone mass during moderate weight loss in older overweight adults with type 2 diabetes? Osteoporos Int 16:1703-1712

21. Dunstan DW, Vulikh E, Owen N, Jolley D, Shaw J, Zimmet P (2006) Community center-based resistance training for the maintenance of glycemic control in adults with type 2 diabetes. Diabetes Care 29:2586-2591

22. Snowling NJ, Hopkins WG (2006) Effects of different modes of exercise training on glucose control and risk factors for complications in type 2 diabetic patients: a meta-analysis. Diabetes Care 29:2518-2527

23. Sayer AA, Dennison EM, Syddall HE, Gilbody HJ, Phillips DI, Cooper C (2005) Type 2 diabetes, muscle strength, and impaired physical function: the tip of the iceberg? Diabetes Care 28:2541-2542

24. Willey KA, Singh MA (2003) Battling insulin resistance in elderly obese people with type 2 diabetes: bring on the heavy weights. Diabetes Care 26:1580-1588

25. Pedersen M, Bruunsgaard H, Weis N et al (2003) Circulating levels of TNF-alpha and IL-6-relation to truncal fat mass and muscle mass in healthy elderly individuals and in patients with type-2 diabetes. Mech Ageing Dev 124:495-502

26. Park SW, Goodpaster BH, Strotmeyer ES et al (2006) Decreased muscle strength and quality in older adults with type 2 diabetes: the health, aging, and body composition study. Diabetes 55:18131818

27. Evans WJ (2004) Protein nutrition, exercise and aging. J Am Coll Nutr 23:601S-609S

28. Vickers KS, Nies MA, Patten CA, Dierkhising R, Smith SA (2006) Patients with diabetes and depression may need additional support for exercise. Am J Health Behav 30:353-362

29. Lindstrom J, Ilanne-Parikka P, Peltonen M et al (2006) Sustained reduction in the incidence of type 2 diabetes by lifestyle intervention: follow-up of the Finnish Diabetes Prevention Study. Lancet 368:1673-1679 